\title{
SPIRITUALITY, QUALITY OF LIFE, DEPRESSION AND DEMENTIA
}

\author{
Océane Agli \\ Department of Psychology, EA 2114, University of Tours (France)
}

\begin{abstract}
This study aimed to observe dementia's role in the relationship between spirituality, quality of life, and depression in aging. The sample included 61 participants between 65 and 98 years old, separated into two groups: participants diagnosed with dementia $(\mathrm{N}=31)$ and control participants $(\mathrm{N}=30)$. There was no significant difference in spirituality between demented and control participants; however, different patterns of correlation were observed between spirituality, depression, and quality of life in these groups. Although the level of spirituality did not differ despite dementia, this pathology would appear to play a role in the relationship between spirituality, quality of life, and depression.
\end{abstract}

Keywords: Dementia, depression, elderly, quality of life, spirituality.

\section{Introduction}

According to Rivier, Hongler, and Sutter (2008), spirituality is a human cognitive approach which seeks to give meaning to life, to set values, and sometimes to seek transcendence, resulting in a spiritual identity. This is part of human development, especially in adults and the elderly. For Dalby (2006), spirituality corresponds to a search for meaning and purpose at a time of life when earlier sources of meaning and purpose may be diminishing. Several studies have highlighted that positive effects of spirituality on mental health (Ellison \& Fan, 2008). In the elderly these benefits can also be observed, on quality of life and wellbeing (Koenig, 1994), stress (Levin, 1996) and depression (Koenig et al., 1992).

To evaluate spirituality, Peterman, Fitchett, Brady, Hernandez, and Cella (2002) developed a brief, reliable, valid measure of spiritual well-being, the Functional Assessment of Chronic Illness Therapy-Spiritual Well-being Scale (FACIT-Sp). A few years later, a short version was created (Canada, Murphy, Fitchett, \& Peterman, 2008): the FACIT-Sp12 item, based on three dimensions: mean, peace, and faith.

When dementia is diagnosed, patients often experience a situation of huge stress, which plunges them into a loss of control and questions their identity. Indeed, dementia is a degenerative neural syndrome characterized by the emergence of neuropsychological deficits, which impact the individual's autonomy. It combines a memory disorder with impairment in other cognitive functions (diagnostic criteria of Diagnostic and Statistical Manual of Mental Disorders-V, 2013). Mood and behavioral disorders (depression, anxiety, psychotic symptoms, agitation, and apathy) are also common. All these symptoms have an impact on the subject's capacity to reason and adapt and disrupt social and relational daily life (Auriacombe \& Orgogozo, 2004). Nevertheless, several studies have shown that dementia patients at a mild to moderate stage (with preserved language skills) are able to correctly estimate their quality of life (Brod, Steward, Sands, \& Walton, 1999).

A systematic review of how spirituality can help to cope with the early stages of Alzheimer's disease has been conducted (Beuscher \& Beck, 2008). Among the six studies reviewed, three emphasized the importance of maintaining contact with family, or even with the environment and nature (Phinney, 1998; Stuckey, Post, Ollerton, FallCreek, \& Whitehouse, 2002). This allows people to connect with their spiritual side, which seems extremely important to improve their quality of life and ability to cope with the disease. A more recent systematic review (Agli, Bailly, \& Ferrand, 2014) underlined the beneficial effects of spirituality among older people with dementia, especially regarding quality of life (Dalby, Sperlinger, \& Boddington, 2012; Jolley et al., 2010; MacKinlay \& Trevitt, 2010). In a longitudinal study, MacKinlay (2012) observed a reduction in levels of depression at a three-month follow-up in participants belonging to a pastoral group. In a study by Stuckey et al. (2002), the authors specified that participants did not focus on the future. Instead, they centered on the present hope, living day by day, to manage better and cope with their disease. 
However, methodological limitations reduce the scope of the results obtained by the studies cited previously: spirituality is often assimilated with religion, the selection criteria are not clearly explained, and the diagnosis of dementia is not always clearly established. On the other hand, if spirituality allows a better quality of life, we do not know if maintaining a good quality of life would allow spiritual development, since no measure of a direct link between spirituality and quality of life has been conducted. The objectives of this study were therefore: (a) to examine whether older people with dementia reported significantly different spirituality scores than the control participants, and (b) to examine the role of dementia in the relationship between spirituality, quality of life, and depression among older people.

\section{Method}

\subsection{Participants}

Sixty-one participants between 65 and 98 years old living in four nursing homes in the Centre region in France were recruited. Participants who had psychotic or addictive disorders (particularly alcoholism) were excluded. The common inclusion criterion was age 65 or older. The experimental design of this study involved dividing the participants into two groups: a test group that represented participants with dementia, and a control group for comparison. The test group $(\mathrm{N}=31)$ consisted of participants diagnosed with dementia (established prior to entering the home and reported in their medical file, or established since entering the institution, through neuropsychological assessments conducted during memory consultations, or using brain scanners). For the control group $(\mathrm{N}=30)$, participants had no dementia pathology. There were 47 women $(77.05 \%)$ and 14 men $(22.95 \%)$. The mean age was 87.23 years old $(\mathrm{SD}=6.48 ; 65-98$ years old $)$.

\subsection{Procedure}

The study protocol was approved by the management of the nursing homes. Each participant was informed that his or her participation in the study was voluntary and anonymous. The families and/or guardians of the participants with dementia were also notified of the progress of the protocol. An information document and a written consent form were signed in duplicate. The protocols were collected by a psychologist, in a hetero-evaluation, in a quiet room. To protect the identity of the participants, data were collected using codes consisting of a combination of letters and numbers.

\subsection{Measures}

To evaluate spirituality, the FACIT-Sp12 (Canada et al., 2008) was used. This scale was recently validated with 11 items on French older people (Agli, Bailly, \& Ferrand, 2017) and can be used on people with cognitive impairment. Participants evaluated items on a 5-point Likert scale (from 0 "not at all" to 4 "very much"). The questionnaire provides four scores: one per dimension and one overall. A high overall score reflects a high level of spirituality. The internal consistency of these subscales is good (Peace $\alpha=.66$; Faith $\alpha=.77$ ), with the exception of the mean dimension, which is just below the threshold $(\alpha=.59)$. The overall scale has good internal consistency $(\alpha=.81)$.

The subjects' cognitive level was evaluated using the Mini Mental State Evaluation (Folstein, Folstein, \& McHugh, 1975). The threshold score for the early stage of dementia is set at 10 . Below this score, cognitive impairment is considered to be too high and can create a bias of understanding instructions and/or language.

To assess quality of life, the French version (Schwebel et al., 2010) of the Dementia Quality of Life scale (DQoL) (Brod et al., 1999) was used. This scale, assessing quality of life for the elderly with dementia, is composed of 29 items divided into five dimensions: self-esteem, positive emotions and humor, negative emotions, the sense of belonging, and aesthetics. A thirtieth item evaluates the overall quality of life (DQoL30): “Overall, how would you rate your quality of life?". A high score reflects a good quality of life. The DQoL has been validated for patients with a minimum score of 12 on the MMSE (Mini-Mental State Examination). In this study, there was good internal consistency for the five subdimensions of the scale (Aesthetics $\alpha=.80$; Sense of belonging $\alpha=.72$; Self-esteem $\alpha=.70$; Positive emotions and humor $\alpha=.82$; Negative emotions $\alpha=.89$ ).

Finally, the level of depression was evaluated with the short version of the Geriatric Depression Scale (Yesavage et al., 1982) and validated in French by Bourque, Blanchard and Vézina (1990). A recent study validated its use with elderly people with cognitive impairment with a MMSE score of 10 (Conradsson et al., 2013). The Cronbach alpha for the current study sample was also good $(\alpha=.74)$. 


\subsection{Data analysis}

To see if spirituality, quality of life, and depression were differently expressed according to cognitive level (presence or absence of dementia), we performed a comparison of means. Data tended not to follow a normal distribution and we therefore conducted a non-parametric Mann-Whitney test on the two independent samples (dementia group and control group). In a second step, rank correlations (measured by the Spearman coefficient) between the dimensions of the DQoL/GDS and the factors of the FACIT were performed. Effect size can be defined as small $(0.3<\mathrm{r}<0.5)$, moderate $(0.5<\mathrm{r}<0.7)$, or large $(0.7<\mathrm{r})$. All analyzes were performed using Statistica V13.3 software.

\section{Results}

\subsection{Comparisons of means of participants with dementia versus controls}

Participants with dementia had a marginally higher depression score $(\mathrm{Z}=1.93, \mathrm{p}=.054)$ than control participants. In addition, it is important to note that participants with dementia reported a score above the threshold of $5(\mathrm{M}=6.45$; $\mathrm{SD}=3.25)$, indicating the presence of mild depression, whereas control participants report a score slightly below this threshold $(M=4.97 ; S D=3.15)$. Participants in the dementia group also reported significantly lower self-esteem scores than control participants $(Z=-2.15$, $\mathrm{p}=.031$ ). Finally, no significant effect was observed on the other dimensions of quality of life, particularly spirituality. In other words, participants with dementia had similar scores of quality of life (sense of aesthetics, sense of belonging, positive and negative emotions, and overall quality of life) and spirituality (mean, peace, faith and general spirituality) to control participants.

\subsection{Links between spirituality, quality of life and depression}

The correlation analyzes between FACIT, DQoL and GDS revealed different patterns of correlation among the demented and control participants. In particular, regarding correlations greater than 0.50 indicating a large effect size, we can observe more significant correlations among control participants than among older people with dementia. Thus, the mean factor was strongly associated with sense of aesthetics $(r=0.69)$, sense of belonging $(r=0.53)$, positive emotions and humor $(r=0.64)$ and the overall item of quality of life $(\mathrm{r}=0.52)$ in the control group. The peace factor was also strongly correlated with four dimensions of DQoL (sense of belonging, self-esteem, positive and negative emotions) in the same group. In contrast, in the dementia group, the mean factor was only strongly correlated with the overall item of quality of life $(r=0.50)$, whereas the peace factor was mainly associated with sense of aesthetics $(r=0.52)$, self-esteem $(r=0.53)$ and positive emotions/humor $(\mathrm{r}=0.59)$. No significant correlation was observed between the faith factor and quality of life in the control group, whereas for participants with dementia two moderate correlations were found with the aesthetic research $(r=0.44)$ and positive emotions/humor $(r=0.45)$.

Regarding the link between spirituality and depression, only the mean factor was negatively correlated (moderate effect size) with depression in the dementia group $(\mathrm{r}=-0.36)$. On the other hand, in the control group, mean $(r=-0.64)$ and peace $(r=-0.56)$ factors were strongly correlated to depression, as well as the overall measure of spirituality $(r=-0.64)$. Finally, it should be noted that the factor faith was not significantly correlated with depression, for either group.

\section{Discussion}

The study objectives were: (a) to examine whether older people with dementia report significantly different spirituality scores from the control participants, and (b) to examine the role of dementia in the relationship between spirituality, quality of life, and depression among older people. First, the results show no significant difference between older people with dementia and control participants concerning spirituality. Indeed, the demented participants had similar scores to the control participants for each dimension (Mean, Peace, Faith) and also for the overall measure (total score FACIT). In other words, dementia does not interfere with the communication of spiritual needs (as long as the person's ability to understand and express oneself is preserved). These results confirm those of MacKinlay and Trevitt (2010). Thus, older people with dementia can rely on their spiritual resources in the same way as older people without cognitive impairment.

On the other hand, among the five dimensions of DQoL, and also the overall quality-of-life item, only the self-esteem dimension presented a significantly different mean from control participants. Thus, although older people with dementia are able to maintain a satisfactory quality of life (Byrne-Davis, Bennett, \& Wilcock, 2006), it appears that they have a more negative judgment of themselves. Because anosognosia occurs only in the advanced stages of dementia, ill people are long aware of their difficulties 
and their loss of capacity. It is possible that this perception is related to their decline in self-esteem and an increasing feeling of being worthless.

In a second analysis, correlations were performed to observe links between spirituality, quality of life, and depression. The results mainly show more significant and strong correlations among control participants. Indeed, in the dementia group, the mean dimension was associated only with the overall quality-of-life item. In other words, the more participants give meaning to their existence, the higher they rate their quality of life. The peace dimension was correlated with three other factors of the DQoL: sense of aesthetics, self-esteem, and positive emotions/humor. This corroborates Trevitt and MacKinlay's (2006) study, which emphasized the preserved abilities of insight and humor in dementia. Thus, the peace dimension correlates more with quality of life than with the mean dimension. The latter is a cognitive dimension more strongly associated with physical health, whereas the former is an emotional factor and is more related to mental health (Canada et al., 2008). This means that older people with dementia seem more concerned about their mental health in relation to their deficits. Finally, two significant moderate correlations were found between the faith dimension and quality of life (sense of aesthetics and positive emotions/humor). On the other hand, among the control participants no significant link was found between this more religious dimension and quality of life, as if realization of the disease revealed a desire to make a bond between religious beliefs and the search for beauty through the environment, which is in line with results in the literature (Phinney, 1998; Stuckey et al., 2002). It should be noted that items corresponding to the sense of aesthetics dimension of the DQoL mainly concern appreciation of nature (3 items out of 5 ).

Regarding the connection between spirituality and depression, more negative correlations were observed in control participants (Peace and Mean), whereas only the mean factor was correlated with depression in participants with dementia. Thus, for older people the stronger the sense of meaning they have in their lives, the less depressed they feel. It is possible that awareness of their pathology has created or reactivated this need for meaning in order to cope better with symptoms and changes in daily life (Auriacombe \& Orgogozo, 2004). That is to say that the faith dimension is not significantly correlated to depression in either group.

Nevertheless, each participant was asked what criteria led to the diagnosis of dementia. In a patient's medical file, it is not uncommon to see the words "neurodegenerative syndrome," but with no documents (results of medical examinations, neuropsychological tests, family testimonials, etc.) attesting to this conclusion. In addition, it is important to note the difficulty sometimes encountered in differentiating between the diagnosis of dementia and other pathologies. Furthermore, when a probable diagnosis is made, the choice of the MMSE - which remains universal - to determine the stage of progression of the disease (mild, moderate, or advanced stage) could be questioned. In fact, non-demented older people may fail this test, and older people with dementia may succeed. Dementia syndromes may differ according to the time of day (morning/evening, after family visit, before meal, etc.), the place (home, institution, hospital, etc.), the person (personality, mood, etc.), the interlocutors and how they are perceived, or the family. It should also be noted that the small sample in our study represents a limit because it is not necessarily representative of the population, and is insufficient for highly developed statistical analysis. It could thus be interesting to conduct a similar study, but this time analyzing by age group, considering the wide age range (from 65 to 98 years) in our study.

In conclusion, in this study different patterns of correlation are observed between demented and control older people concerning the relationship between spirituality, quality of life, and depression. It would be interesting to continue this research in terms of concrete applications. Thus, spirituality trajectories could be observed in the same way as changes in individual dementia syndromes and quality of life. Finally, if the spirituality concept persists in pathological aging, it would also be interesting to study what happens in advanced stages of neurodegenerative diseases, when language abilities are reduced and understanding is impaired.

\section{References}

Agli, O., Bailly, N., \& Ferrand, C. (2014). Spirituality/religion in the elderly with dementia: A systematic review. International Psychogeriatrics, 27(5), 715-725. doi:10.1017/ S1041610214001665

Agli, O., Bailly, N., \& Ferrand, C. (2017). Validation of the functional assessment of chronic illness therapy - spiritual well-being (FACIT-Sp12) on French old people. Journal of Religion and Health, 56(2), 464-476. doi:10.1007/s10943-016-0220-0

American Psychiatric Association. (2013). DSM-5: diagnostic and statistical manual of mental disorders (5th edition). Washington D.C: American Psychiatric Association

Auriacombe, S., \& Orgogozo, J. M. (2004). Syndrome démentiel. [Demential syndrome]. EMC-Neurologie, 1(1), 55-64. doi:10.1016/j.emcn.2003.10.003 
Beuscher, L., \& Beck, C. (2008). A literature review of spirituality in coping with early-stage Alzheimer's disease. Journal of Clinical Nursing, 17(5a), 88-97. doi:10.1111/j.13652702.2007.02126.x

Bourque, P., Blanchard, L., \& Vezina, J. (1990). Étude psychométrique de l'Échelle de dépression gériatrique. [Psychometric study of the Geriatric Depression Scale]. Revue Canadienne Du Vieillissement, 9(4), 348-355. doi:10.1017/S0714980800007467

Brod, M., Steward, S. L., Sands, L., \& Walton, P. (1999). Conceptualizations and measurement of quality of life in dementia: The dementia quality of life instrument (DQoL). The Gerontologist, 39(1), 25-35. doi:10.1093/geront/39.1.25

Byrne-Davis, L. M., Bennett, P. D., \& Wilcock, G. K. (2006). How are quality of life ratings made? Toward a model of quality of life in people with dementia. Quality of Life Research, 15(5), 855-865. doi:10.1007/s11136-005-5416-9

Canada, A. L., Murphy, P. E., Fitchett, G., Peterman, A., \& Schover, L. R. (2008). A 3-factor model for the FACIT-Sp. Psycho-Oncology, 17(9), 908-916. doi:10.1002/pon.v17:9

Conradsson, M., Rosendahl, R., Littbrand, H., Gustafson, Y., Olofsson, B., \& Lövheim, H. (2013). Usefulness of the geriatric depression scale 15-item version among very old people with and without cognitive impairment. Aging and Mental Health, 17(5), 638-645. doi: $10.1080 / 13607863.2012 .758231$

Dalby, P. (2006). Is there a process of spiritual change or development associated with ageing? A critical review of research. Aging and Mental Health, 10(1), 4-12. doi:10.1080/ 13607860500307969

Dalby, P., Sperlinger, D. J., \& Boddington, S. (2012). The lived experience of spirituality and dementia in older people living with mild to moderate dementia. Dementia: the International Journal of Social Research and Practice, 11(1), 75-94. doi:10.1177/ 1471301211416608

Ellison,C.G.,\&Fan,D.(2008).Dailyspiritualexperiencesandpsychologicalwell-beingamong us adults. Social Indicators Research, 88(2), 247-271. doi:10.1007/s11205-007-9187-2

Folstein, M. F., Folstein, S. E., \& McHugh, P. R. (1975). Mini-Mental State: A practical method for grading the cognitive state of patients for the clinician. Journal of Psychiatric Research, 12(3), 189-198. doi:10.1016/0022-3956(75)90026-6

Jolley, D., Benbow, S. M., Grizzell, M., Willmott, S., Bawn, S., \& Kingston, P. (2010). Spirituality and faith in dementia. Dementia: The International Journal of Social Research and Practice, 9(3), 311-325. doi:10.1177/1471301210370645

Koenig, H. G., Cohen, H. J., Blazer, D. G., Pieper, C., Meador, K. G., Shelp, F., ... DiPasquale, B. (1992). Religious coping and depression among elderly, hospitalized medically ill men. American Journal of Psychiatry, 149(12), 1693-1700. doi:10.1176/ajp.149.12.1693

Levin, J. S. (1996). How religion influences morbidity and health: Reflections on natural history, salutogenesis and host resistance. Social Science Medicine, 43(5), 849-864. doi:10.1016/02779536(96)00150-5

MacKinlay, E. (2012). Resistance, resilience, and change: The person and dementia. Journal of Religion, Spirituality \& Aging, 24(1-2), 80-92. doi:10.1080/15528030.2012.633048

MacKinlay, E., \& Trevitt, C. (2010). Living in aged care: Using spiritual reminiscence to enhance meaning in life for those with dementia. International Journal of Mental Health Nursing, 19(6), 394-401. doi:10.1111/j.1447-0349.2010.00684.x

Peterman, A. H., Fitchett, G., Brady, M. J., Hernandez, L., \& Cella, D. (2002). Measuring spiritual well-being in people with cancer: The functional assessment of chronic illness therapy-Spiritual well-being scale (FACIT-Sp). Annals of Behavioral Medicine, 24(1), 49-58. doi:10.1207/S15324796ABM2401_06

Phinney, A. (1998). Living with dementia from the patient's perspective. Journal of Gerontological Nursing, 24(6), 8-15. doi:10.3928/0098-9134-19980601-06

Rivier, C., Hongler, T., \& Suter, C. (2008). La spiritualité en soins palliatifs. [Spirituality in palliative care]. Guide Des Soins Palliatifs Du Medecin Vaudois, 6(5), 1-26.

Schwebel, G., Dramé, M., Jolly, D., Boyer, F., Morrone, I., Di Pollina, L., ... Novella, J. L. (2010). Validation psychométrique du questionnaire dementia quality of life (DQoL) en langue française. [Psychometric validation of the dementia quality of life questionnaire in French language]. La Revue De Gériatrie, 35(2), 93-100.

Stuckey, J. C., Post, S. G., Ollerton, S., FallCreek, S. J., \& Whitehouse, P. J. (2002). Alzheimer's disease, religion and the ethics of respect for spirituality a community dialogue. Alzheimer's Care Quarterly, 3(3), 199-207.

Trevitt, C., \& MacKinlay, E. (2006). "I am just an ordinary person...": Spiritual reminiscence in older people with memory loss. Journal of Religion, Spirituality \& Aging, 18(2-3), 79-91. doi:10.1300/J496v18n02_07

Yesavage, J. A., Brink, T. L., Rose, T. L., Lum, O., Huang, V., Adey, M., \& Leirer, V. O. (1982). Development and validation of a geriatric depression screening scale: A preliminary report. Journal of Psychiatric Research, 17(1), 37-49. doi:10.1016/0022-3956(82)90033-4 